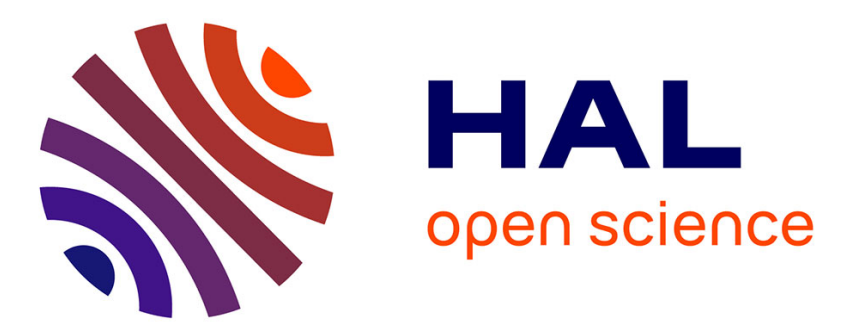

\title{
Refonder le Conseil supérieur de la magistrature dans la Tunisie post-Ben Ali : corporatismes juridiques et nouveaux arrangements institutionnels
}

Eric Gobe

\section{- To cite this version:}

Eric Gobe. Refonder le Conseil supérieur de la magistrature dans la Tunisie post-Ben Ali: corporatismes juridiques et nouveaux arrangements institutionnels. Droit et Société, 2019, Le droit à l'épreuve des algorithmes, 3 (103), pp.629-648. 10.3917/drs1.103.0629 . halshs-02424107

\section{HAL Id: halshs-02424107 \\ https://shs.hal.science/halshs-02424107}

Submitted on 26 Dec 2019

HAL is a multi-disciplinary open access archive for the deposit and dissemination of scientific research documents, whether they are published or not. The documents may come from teaching and research institutions in France or abroad, or from public or private research centers.
L'archive ouverte pluridisciplinaire HAL, est destinée au dépôt et à la diffusion de documents scientifiques de niveau recherche, publiés ou non, émanant des établissements d'enseignement et de recherche français ou étrangers, des laboratoires publics ou privés. 


\title{
Refonder le Conseil supérieur de la magistrature dans la Tunisie post-Ben Ali : corporatismes juridiques et nou- veaux arrangements institutionnels
}

\author{
Éric Gobe
}

Institut de recherches et d'études sur le monde arabe et musulman - Maison méditerranéenne des sciences de l'homme (IREMAM/MMSH), 5 rue du Château de l'horloge, F-13090 Aix-en-Provence.

<gobe@mmsh.univ-aix.fr>

Trois ans après la chute du régime autoritaire de Ben Ali, le 26 janvier 2014, la nouvelle constitution tunisienne est adoptée dans l'euphorie à une écrasante majorité par les députés de l'Assemblée nationale constituante (ANC) 1. L'État de droit et l'indépendance du pouvoir juridictionnel y sont énoncés. Le texte constitutionnel est globalement bien reçu par les institutions internationales gardiennes des standards constitutionnels internationaux en matière de démocratie et de droits humains : la Commission européenne pour la démocratie par le droit (commission de Venise) salue certaines avancées d'un texte protecteur des justiciables (proclamation du principe du procès équitable, obligation de neutralité du juge, publicité des audiences, etc.) et des magistrats (indépendance, immunité, principe de l'inamovibilité du juge, etc.).

Ce dispositif est garanti par l'institutionnalisation d'un Conseil supérieur de la magistrature (CSM) 2 qui, selon l'article 114 de la Constitution, «veille au bon fonctionnement de la justice et au respect de son indépendance ». La commission de Venise se félicite d'ailleurs qu'une section spécifique de la constitution lui soit consacrée : elle y voit l'expression « du choix du constituant tunisien de confier la supervision du système judiciaire à un organe indépendant composé en grande partie par des juges élus par leurs pairs » 3 . Toutefois, elle émet des réserves sur la complexité

1. Cet article s'inscrit dans le cadre des résultats du projet de recherche ERC TARICA. Il a reçu le soutien du European Research Council (ERC) à travers le programme européen Horizon 2020 $\left(n^{\circ} 695674\right)$.

2. Le CSM sous Bourguiba et Ben Ali était une instance soumise au pouvoir présidentiel. Composé de neuf magistrats nommés et quatre élus, il avait à sa tête le président de la République (président) et le ministre de la Justice (vice-président). Voir Mohamed Salah BEN AÏSSA, « La compétence exclusive du Conseil supérieur de la magistrature en matière disciplinaire ou... quand le Conseil constitutionnel brise la jurisprudence du tribunal administratif », in Hommage à Dali JAZI, La Manouba (Tunisie), CPU, 2010, p. 151-181.

3. COMMISSION EUROPEENNE POUR LA DEMOCRATIE PAR LE DROIT (COMMISSION DE VENISE), Observations sur le projet final de la Constitution de la république tunisienne, Strasbourg, 17 juillet 2013, avis 733/2013, $\quad \operatorname{cdl}(2013) 034, \quad$ [en ligne : <http://www.venice.coe.int/webforms/documents/?pdf=cdl(2013)034-f>]. 
de l'architecture institutionnelle du CSM 4 et regrette que ses compétences concernant les relations entre les différents conseils, ainsi que la carrière et la discipline des magistrats, soient formulées en termes très généraux et renvoyées à l'adoption d'une loi organique. La Constitution laisse ainsi une importante « zone grise » 5 quant à la place du CSM dans l'organisation juridictionnelle.

Les deux professions les plus prestigieuses de l'arène judiciaire, magistrats et avocats, vont s'emparer de cette zone grise pour faire valoir leur référentiel 6 de l'organisation et des missions du CSM auprès du ministre de la Justice et des députés de l'Assemblée des représentants du peuple (ARP). L'élaboration de la loi organique sur le CSM est précisément le résultat de processus politiques et professionnels dans lesquels ont été impliqués des «acteurs multiples [poursuivant] des logiques d'action hétérogènes $» 7$.

Elle a cristallisé, dans un moment de construction de l'architecture institutionnelle d'un nouveau régime, des conflits professionnels et politiques hérités du pouvoir autoritaire de Ben Ali et de la « conjoncture politique fluide » 8 traversée par la Tunisie. Aussi, des éléments de «l'ancien régime», «notamment leurs formes et ressources organisationnelles [...], liens de solidarité et capitaux sociaux, habitus ou routine des acteurs » 9 sont-ils présents dans la discontinuité institutionnelle tunisienne. Autrement dit, certains cadres cognitifs et normatifs, construits sous la présidence de Ben Ali, demeurent prégnants. Ceux-ci ont un impact sur la manière dont l'action publique et la légitimité professionnelle des corporations juridiques s'articulent dans la Tunisie post-Ben Ali 10.

4. Le CSM est formé de quatre organes, les trois conseils respectifs des justices judiciaire, administrative et financière, auquel s'ajoute une assemblée plénière desdits conseils.

5. Jean-Philippe BRAS, «De l'État légal à l'État de droit? Le statut constitutionnel de la justice au Maghreb », p. 91, in Éric GoBE (dir.), Des justices en transition dans le monde arabe ? Contributions à une réflexion sur les rapports entre justice et politique, Rabat: CJB, coll. « Description du Maghreb», Open Edition, 2016 [en ligne : <http://books.openedition.org/cjb/753>].

6. Entendu ici dans le sens du spécialiste des politiques publiques Pierre Muller comme un «cadre d'interprétation » idéelle sectorielle de ce que doit être, dans ce cas d'espèce, la régulation de la justice par le CSM. Pierre MULLER, «L'analyse cognitive des politiques publiques : vers une sociologie politique de l'action publique », Revue française de science politique (RFSP), 55 (1), 2000.

7. Jacques COMmaille, Une sociologie politique de la carte judiciaire, Paris : PUF, 2000, p. 122.

8. Michel DoBry, Sociologie des crises politiques. La dynamique des mobilisations multisectorielles, Paris : Presses de Sciences Po, 1986.

9. Toutefois, comme le note Michel DOBRY, «Les voies incertaines de la transitologie : choix stratégiques, séquences historiques, bifurcations et processus de path dependence », RFSP, 50 (4-5), 2000, p. 594 : ces « "survivances" éclatées » ne nous disent pas grand-chose sur «les voies d'extrication du système préexistant, elles-mêmes conçues comme "dépendantes" des points de départ des processus » qui sont au cœur des approches de path dependance.

10. Nous comprenons la légitimité professionnelle comme « le produit de la rencontre entre des stratégies, mises en œuvre par des organisations ou des institutions pour se rendre crédibles et acceptables aux yeux de leur public et un environnement social et [politique] plus ou moins favorable, perméable à ces stratégies » (Patrice DURAN et Thomas LE BIANIC, «Introduction générale », in Thomas LE BIANIC et Antoine VION (dir.), Action publique et légitimités professionnelles, Paris : LGDJ, 2008, p. 27-28). 
Pour reprendre la formulation de Sara Dezalay 11, l'action des professionnels du droit révèle «l'encastrement des champs juridiques dans les champs nationaux du pouvoir d'État ». L'analyse des débats et mobilisations autour du texte relatif au CSM permet de montrer précisément combien les espaces sociaux juridique et politique «sont liés par une relation de "co-production" » 12 .

En effet, le moment d'élaboration du texte de loi relatif au CSM (2015-2016) a mis principalement aux prises des professions juridiques, à travers leurs instances représentatives, et des acteurs politiques (les parlementaires), eux-mêmes largement issus de l'un des groupes professionnels du secteur de la justice (les avocats) 13 . Aussi nous intéressons-nous à l'action juridico-politique des structures professionnelles représentant avocats et magistrats.

Dans le cas présent, cette approche, bien que biaisée (nous ne prenons pas en compte les non-engagés et les éventuelles fractions dissidentes de la profession), nous apparaît légitime dans la mesure où les organisations professionnelles de magistrats et d'avocats ont été, pour l'occasion, les seuls interlocuteurs reconnus par les gouvernants et que nous n'avons pas repéré de professionnels du droit « réfractaires » prenant la parole pour contester publiquement les prétentions de leurs représentants « de parler et d'agir en leur nom ») 14 .

Pour appréhender les enjeux autour de la création du CSM, nous nous centrerons sur l'action des corporatismes professionnels, c'est-à-dire des groupes structurés «agissant autour d'une culture de métier dans laquelle [leurs] membres se reconnaissent, et à laquelle ils s'identifient prioritairement. » 15 .

Précisément, les structures professionnelles ont mobilisé diverses ressources et moyens d'action tant dans des arènes institutionnelles que dans d'autres espaces sociaux (notamment la « rue » et la presse) pour faire prévaloir des représentations et des croyances (c'est-à-dire « une culture de métier » pour reprendre la formulation de

11. Sara DEZALAY, «Les juristes en Afrique : entre trajectoires d'État, sillons d'empire et mondialisation », p. 5-23, p. 13, in ID. (dir.), dossier « Juristes faiseurs d'État », Politique africaine, 138, 2015.

12. George H. KAREKWAIVANANE, « Les juristes entre "africanisation" et transition politique », in ibid., p. $49-70$, p. 51.

13. Pour conduire cette recherche, nous avons mené des entretiens avec l'ancien ministre de la Justice du premier gouvernement Essid, ainsi qu'avec les dirigeants des principales organisations de magistrats et d'avocats qui, en raison de leur fonction de porte-parole, nous ont donné l'autorisation de les citer. Nous avons également exploité les archives de diverses institutions (journaux, organisations professionnelles, commission de législation de l'Assemblée des représentants du peuple et ministère de la Justice).

14. Lucien KARPIK, «Les professions libérales sont-elles solubles dans le marché », in Thomas LE BIANIC et Antoine VION (dir.), Action publique et légitimités professionnelles, op. cit., p. 279-288, p. 284.

15. Jacques CAPdevielle, Modernité du corporatisme, Paris : Presses de Science Po, 2001, p. 9. Nous n'utilisons pas le terme corporatisme tel que défini par les doctrines mobilisées par le régime de Vichy et le fascisme italien qui, s'appuyant sur les organisations professionnelles, développaient une conception organique de l'ordre social refusant tant le conflit que l'idée de lutte des classes. Nous ne faisons pas non plus référence aux travaux de Philippe SCHMITTER ( « Still the Century of Corporatism? », The Review of Politics, 36 (1), 1974, p. 85-131) et de ses épigones qui, dans les années 1970, ont mobilisé, par opposition au corporatisme autoritaire, les concepts de corporatisme « sociétal », « libéral », « démocratique » ou «néo-corporatiste ». Celui-ci se caractérisait par l'existence d'organisations sociales, autonomes à l'origine, lesquelles « reconnues et consolidées» par l'État conduisent à travers « un processus d'osmose » avec ce dernier des politiques concertées (Bruno JOBERT et Pierre MULLER, L'État en action : politiques publiques et corporatismes, Paris : PUF, 1987, p. 159-160). 
Jacques Capdevielle) relatives aux fonctions du CSM, mais aussi pour défendre des intérêts et des « privilèges protocolaires » 16 .

Le cadre ainsi posé, peut-on dire avec Terence Halliday et Lucien Karpik 17 que l'action des professions juridiques autour de la refondation du CSM tunisien ressortit à la construction d'un libéralisme politique consacrant les droits individuels et les libertés fondamentales protégeant les citoyens de l'arbitraire de l'État ? A-t-on affaire à un «complexe juridique » de professionnels du droit (principalement les porte-parole du barreau et magistratures), c'est-à-dire à un «système d'interrelations entre acteurs juridiques » 18 en capacité de faire évoluer un régime politique autoritaire vers une démocratie libérale ? Dans le contexte tunisien, la réponse à ces deux questions est mitigée. Sans nier l'existence d'un éthos libéral au sein du barreau, voire dans la magistrature, la démarche de ces deux auteurs a le principal inconvénient, dans le cas d'espèce, de négliger les revendications socioprofessionnelles contradictoires portées par les organisations de juristes confrontées à un passé autoritaire et en prise avec des changements politiques d'ampleur.

À travers l'analyse, d'une part, des interactions des professionnels du droit entre eux et, d'autre part, de leurs rapports avec les gouvernants, nous nous proposons de montrer que le texte voté par le Parlement est largement l'expression de la crainte d'une autonomie professionnelle trop grande des magistrats, présente chez une partie des parlementaires, du Gouvernement et au sein d'un barreau lui-même pourvoyeur d'élus politiques. La loi est ainsi le résultat de rapports conflictuels entre l'avocature, minorée sous Ben Ali, les représentants de la magistrature dont certains de ses membres ont été les instruments d'une justice mise au service du régime autoritaire, des élites islamistes violemment réprimées par Ben Ali et des représentants de l'ancien régime revenus à la tête de l'État, après les élections présidentielles et législatives de 2014.

\section{Les corporatismes juridiques et la question du Conseil supérieur} de la magistrature

Le 31 janvier 2015, Hafedh Ben Salah, ministre de la Justice d'un Gouvernement en fin de mandat ${ }^{19}$, préside une journée d'information où sont invités trois organisations professionnelles de magistrats et l'Ordre national des avocats de Tunisie (ONAT) pour exposer le contenu de l'avant-projet de loi organique relatif à la création du CSM.

Le temps presse, plus de trois mois se sont écoulés depuis les élections législatives, alors que les dispositions transitoires de la constitution précisent que le CSM doit être mis en place dans un délai maximum de six mois à compter de la date desdites élections. Par conséquent, le texte doit être introduit rapidement dans l'agenda

16. Alain BANCAUD, « Normalisation d'une innovation : le conseil supérieur de la magistrature sous la IV $^{\mathrm{e}}$ République », Droit et Société, 63-64, 2006, p. 372.

17. Terence C. HALLIDAY et Lucien KARPIK (dir.), Lawyers and the Rise of Political Liberalism. Europe and North America from the Eighteenth to Twentieth Centuries, Oxford : Clarendon Press, 1997.

18. Lucien KARPIK, «Les professions libérales sont-elles solubles dans le marché ? », op. cit., p. 282.

19. Le Gouvernement dit de «technocrates » chargé d'organiser, après la promulgation de la nouvelle Constitution (27 janvier 2014), les élections législatives et présidentielles avant la fin de 2014. 
de l'Assemblée des représentants du peuple (ARP) pour y être adopté. L'enjeu principal est de reconstruire une institution dévoyée sous les présidences de Ben Ali et de Bourguiba. Le CSM est supposé s'insérer dans «le processus d'institutionnalisation d'une "nouvelle justice" » 20 et renvoie à la mise en place d'une nouvelle légitimité pour l'institution juridictionnelle.

L'avant-projet gouvernemental est présenté par le ministre de la Justice comme un texte rédigé « par les représentants des instances juridictionnelles, loin des tiraillements politiques et des surenchères syndicales » 21 . Or, c'est précisément là où le bât blesse pour l'ONAT, l'Association des magistrats tunisiens (AMT), le Syndicat des magistrats tunisiens (SMT) et l'Union des magistrats administratifs (UMA), même si le ministre conclut son intervention en précisant que les propositions avancées par les organisations professionnelles seront prises en considération par la commission.

\section{I.1. Les avocats et le CSM : la revendication d'une légitimité historique}

La vision de l'ONAT relative à l'organisation et les compétences du CSM est nourrie par les contentieux professionnels qui ont marqué l'histoire des relations entre avocats et magistrats judiciaires depuis l'indépendance. Dans la mesure où les gouvernants tunisiens ont considéré la justice comme un instrument de coercition des attitudes politiques dissidentes, les rapports entre les deux principaux corps de l'institution judiciaire ont souvent été conflictuels et empreints de méfiance.

Par ailleurs, sous Ben Ali, le « bas barreau tunisien », c'est-à-dire la fraction majoritaire de la profession, à la fois dominée économiquement et politiquement, a particulièrement mal vécu la massification de la profession : de 1991 à 2011, l'effectif du barreau a été multiplié par près de six, passant d'environ 1400 à 7759 membres et a connu sa plus forte accélération entre 2008 et 2011 avec l'inscription au tableau de l'ONAT de 1500 avocats 22. Ce «bas barreau » et les porte-parole de la profession y ont vu une politique délibérée du pouvoir visant à «affamer» la profession. Les facilités données par le régime autoritaire de Ben Ali aux magistrats d'accéder au barreau, ainsi que la redéfinition des normes professionnelles dans le sens d'une restriction de l'autonomie de l'avocature, notamment dans ses rapports avec les juges (absence d'immunité de l'avocat plaidant devant un tribunal), ont contribué à faire du «magistrat», aux yeux de l'« avocat», un ennemi de la défense judiciaire 23 .

20. La formule est d'Antoine VAUCHEZ, L'institution judiciaire remotivée. Le processus d'institutionnalisation d'une "nouvelle justice» en Italie (1960-2000), Paris: LGDJ, 2004, et fait référence à l'expérience de l'opération mains propres dans l'Italie des années 1990.

21. «Le projet de loi de création du CSM en débat » (en arabe), Le Maghreb, $1^{\mathrm{er}}$ février 2015.

22. La profession compte au 30 juin 2017 un peu plus de 9800 avocats, stagiaires compris. Voir ONAT, Rapport moral 2016-2017. Éric GoBE, Les avocats en Tunisie de la colonisation à la révolution (18832011). Sociohistoire d'une profession politique, Tunis : IRMC, Paris : Karthala, 2013.

23. Éric GoBE, «Penser les relations avocats-magistrats dans la Tunisie indépendante : conflictualité professionnelle et dynamique politique », in Sara DEZALAY (dir.), dossier « Juristes faiseurs d'État », op. cit., p. 115-134. 
Or, la chute du régime de Ben Ali donne l'occasion aux instances ordinales (le bâtonnier et le conseil de l'Ordre) d'obtenir de la part du Gouvernement provisoire une réorganisation du barreau dans le sens d'un rehaussement de son statut aux dépens d'autres professions juridiques 24 . Le barreau a tenté d'imposer un nouveau rapport de force avec la magistrature : rédigé par les instances ordinales, le texte initial du décret-loi relatif à l'avocature restreint l'accès des magistrats à la profession, accorde l'immunité pénale à l'avocat qui plaide (article 47) et exige qu'en cas de poursuites pénales contre un avocat, le président de la section régionale de l'ONAT en soit avisé (article 46).

$\mathrm{Si}$, après une série de grèves menées par la magistrature, le Gouvernement provisoire de Béji Caïd Essebsi biffe dans la version finale du décret-loi relatif à la profession d'avocat les conditions d'accès restrictives imposées aux magistrats, les articles 46 et 47 sont maintenus dans leur rédaction initiale. Depuis 2011, les controverses entre avocats et magistrats autour de l'application du décret-loi enveniment leurs rapports. Au cours de ces dernières années, les incidents d'audience se sont multipliés, ce qui a contribué à alimenter des cycles de grèves et de contre-grèves de la part des deux professions juridiques 25 .

Pour les représentants du barreau, la création du CSM doit donner l'occasion à la profession de ne pas laisser la bride sur le cou des magistrats et de valoriser les gains professionnels acquis à la suite de la participation des avocats au soulèvement de 2010-2011. La forte présence d'avocats au sein de l'ANC avait d'ailleurs permis au barreau de voir consacrer sa revendication d'égalité vis-à-vis de la magistrature, à travers l'article 105 de la Constitution, constitutionnalisant la formulation de l'article 1 du décret-loi d'août 2011 relatif à la profession 26 . De ce point de vue, la première version du texte relatif au CSM ne satisfait pas l'ONAT : ses dispositions concernant sa composition ont l'inconvénient de ne pas mentionner les avocats. Le texte se contente de reprendre la formulation de la Constitution précisant que les trois conseils juridictionnels du CSM se composent d'un « tiers de non-magistrats indépendants choisis parmi les spécialistes ». S'appuyant sur l'article 105 de la Constitution, le bâtonnier revendique la présence explicite d'avocats parmi les membres non-magistrats du CSM 27. À cet argumentaire, les instances ordinales ajoutent la légitimité historique d'un barreau défenseur des droits et des libertés sous la dictature.

Les représentants de l'Ordre véhiculent un référentiel plutôt restrictif des attributions du CSM et revendiquent la mise en place d'un CSM axé sur la carrière et la

24. « Décret-loi $\mathrm{n}^{\circ} 2011-79$ du 20 août 2011 portant organisation de la profession d'avocat », JORT, 23 août 2011, p. 1595-1606.

25. Éric GoBE, «Penser les relations avocats-magistrats dans la Tunisie indépendante : conflictualité professionnelle et dynamique politique », article cité.

26. L'article 105 dispose que : «La profession d'avocat est une profession libre et indépendante, qui participe à la réalisation de la justice et à la défense des droits et libertés. L'avocat bénéficie des garanties légales qui lui assurent une protection et lui permettent l'exercice de ses fonctions. »

27. Entretien avec un ancien bâtonnier de l'ONAT, 2 juillet 2016. L'ONAT propose une composition du tiers des non-magistrats, dans les conseils du CSM, largement dominée par les avocats : un total de onze avocats sur dix-sept membres non-magistrats. 
discipline des magistrats inspiré du modèle français. Or, le premier texte gouvernemental prévoit la prise en charge d'une partie de l'administration du système judiciaire par le CSM qui superviserait plus particulièrement l'inspection générale des affaires juridictionnelles 28 . Ce faisant, les instances ordinales considèrent que le projet gouvernemental oblitère « tout mécanisme de contrôle sur un pouvoir juridictionnel qui supervise tout et n'est contrôlé par personne » 29 . Il convient, par conséquent, que l'inspection reste dans le giron du ministère de la Justice.

D'autres propositions du barreau vont dans le sens d'un amoindrissement des attributions du CSM : il souhaite que l'Institut supérieur de la magistrature (l'école de la magistrature) reste sous la tutelle du ministère de la Justice, tout comme le recrutement des magistrats. Pour résumer en une ligne la position de l'ONAT : un CSM aux compétences larges et dominées par les juges est inenvisageable pour les représentants du barreau.

\section{I.2. Les magistrats et le CSM : un corporatisme fragmenté}

Les organisations professionnelles de magistrats, quant à elles, se retrouvent autour d'un référentiel commun : autorité constitutionnelle et incarnation du pouvoir juridictionnel, le CSM doit être à même de garantir l'indépendance de la fonction de juger en disposant de compétences relatives à la nomination, la carrière et à la discipline des magistrats. Leurs divergences et nuances se rapportent à la composition du CSM et au spectre plus ou moins large de ses attributions en matière d'inspection et de gestion de l'appareil juridictionnel. Mais par-delà les différences de positionnement vis-à-vis du fonctionnement du CSM, leurs conflits sont, d'une part, le résultat de l'histoire du rapport de la magistrature au politique sous le régime autoritaire de Ben Ali et, d'autre part, le fruit d'une « querelle d'audiences » 30.

On fera ici nôtre l'hypothèse selon laquelle « les clivages au sein de la magistrature [tunisienne] sont des clivages autour des audiences ». Cette notion, qui désigne à l'origine « le public des juges à travers leurs décisions judiciaires ou leur comportement individuel »31, est élargie par Samer Ghamroun « aux mobilisations collectives des juges et à leurs actions extra-juridictionnelles »32. Dans une conjoncture tunisienne où les magistrats «se divisent à travers leurs publics » les organisations représentatives jouent « un rôle déterminant dans l'évolution de ces clivages et dans la capacité de chaque courant judiciaire à se maintenir et à se développer, ainsi que dans la capacité des juges à se constituer en interlocuteurs de poids face au pouvoir

28. Ministere DE LA JUSTICE, Projet de loi organique organisant le CSM (en arabe), [en ligne : <http://www.e-justice.tn/fileadmin/fichiers_site_arabe/actualites/projet_loi_csm.pdf].

29. Déclaration d'Ameur Meherzi, président de la section de Tunis de l'ONAT au journal Le Maghreb, « La section des avocats de Tunis et le projet de CSM », 19 février 2015.

30. Samer Ghamroun, « À qui s'adressent les juges ? Les magistrats tunisiens et égyptiens face aux aléas de la représentation professionnelle », in Éric GOBE (dir.), Des justices en transition dans le monde arabe? Contributions à une réflexion sur les rapports entre justice et politique, op. cit., p. 177.

31. Judicial audiences en anglais (notion forgée par la sociologue du droit Laurence Baum). La traduction d'audience par auditoire, nous paraît plus pertinente.

32. Samer GHAmroun, « À qui s'adressent les juges ? Les magistrats tunisiens et égyptiens face aux aléas de la représentation professionnelle », op. cit., p. 172. 
exécutif ». Autrement dit, à travers l'usage de cette notion, il s'agit de répondre à une série de questions : «À qui s'adressent les juges lorsqu'ils se mobilisent? Dans quelles arènes le font-ils ? Avec quelles alliances 33 ?»

Force est de constater que l'AMT d'une part, le SMT et l'UMA d'autre part, ne s'adressent pas aux mêmes « audiences » ou auditoires. Ce clivage est largement issu de la crise de 2005 durant laquelle la magistrature s'était divisée sur la conduite à tenir face à la politique répressive du pouvoir vis-à-vis des avocats militants. Un regard rapide sur l'histoire de l'AMT, l'organisation de magistrats la plus ancienne de la scène judiciaire tunisienne, est nécessaire pour comprendre les positionnements professionnels et politiques des magistrats après la fuite de Ben Ali, ainsi que le caractère tendu des relations entre l'AMT et les deux autres structures professionnelles.

Après 2011, l'AMT a constitué le groupement de magistrats le plus offensif dans la dénonciation du régime autoritaire de Ben Ali. Revendiquant, en 2018, 1600 adhérents, dont une centaine de magistrats des ordres administratifs et financiers, l'AMT a vu le jour en 1988, un an après la prise du pouvoir par le président Ben Ali, dans une conjoncture de relative ouverture du régime autoritaire. Elle n'a guère fait parler d'elle dans les années 1990, période d'affermissement du nouveau pouvoir autoritaire. En 2004, l'élection au bureau exécutif d'une majorité revendiquant l'indépendance de la magistrature vis-à-vis de l'exécutif a contribué à tendre les relations entre l'AMT et les gouvernants. Le dénouement de cette phase de tension s'est déroulé une année plus tard, en mars 2005, lorsque le bureau exécutif a exprimé son opposition à l'intervention des forces de police dans l'enceinte du palais de justice à l'occasion de l'arrestation de l'avocat et opposant politique, Mohamed Abbou.

Le régime de Ben Ali a réagi par la coercition en mutant les membres du bureau exécutif loin de la capitale et en incitant les magistrats qui lui étaient acquis à évincer l'équipe dirigeante de l'AMT au moyen d'un congrès extraordinaire électif 34. Cet épisode fondateur permet à la direction actuelle de l'AMT de se prévaloir de la « légitimité politique tirée de la résistance déployée par les membres du bureau de l'AMT en 2005 face aux pratiques répressives de Ben Ali » 35. Il est également une clé pour comprendre l'«auditoire judiciaire» élargi de l'AMT: l'organisation s'adresse à l'ensemble des citoyens et/ou justiciables tunisiens qu'il convient d'interpeller à travers les médias ou par des actions collectives qui empruntent à un répertoire d'action renvoyant à la grève des audiences, ainsi qu'aux sit-in devant les tribunaux, le Parlement ou le ministère de la Justice.

À l'opposé de l'AMT, le SMT, porteur d'une forme de conformisme vis-à-vis du pouvoir politique, s'adresse à un auditoire judiciaire étroit. Il s'est d'ailleurs consti-

\section{Ibid.}

34. Sana BEN ACHOUR, «La féminisation de la magistrature en Tunisie entre émancipation féminine et autoritarisme politique », in Yadh BEN ACHOUR et Éric GOBE (dir.), Justice, politique et société. L'Année du Maghreb 2005-2006, Paris, CNRS Éditions, p. 55-74).

35. Samer GHAmRoun, «À qui s'adressent les juges ? Les magistrats tunisiens et égyptiens face aux aléas de la représentation professionnelle », op. cit., p. 179. 
tué, en mars 2011, contre l'AMT et ses revendications d'épuration de la magistrature par le «peuple révolutionnaire ». La nouvelle organisation a, dès sa naissance, affirmé son refus d'une épuration du corps des juges lui préférant des poursuites individuelles étayées sur des « dossiers instruits par les institutions judiciaires traditionnelles »36. Revendiquant 1350 adhérents (exclusivement des magistrats judiciaires), le SMT met en avant la nécessité d'éviter « une politisation excessive » de l'action des organisations professionnelles (sous-entendue, celle de l'AMT) et de la recentrer sur la défense des « intérêts matériels et moraux » des magistrats. La structure syndicale, opposée à la forme juridique de l'association, est présentée par ses dirigeants comme la mieux à même de négocier avec les autorités de / pour tout ce qui relève des questions professionnelles. Par ailleurs, le SMT valorise le « dialogue constructif avec les autorités »; et promeut une modération dans l'utilisation de son répertoire d'action (usage de la grève des audiences en dernier recours).

L'AMT n'a pas manqué d'accuser le SMT de regrouper les magistrats proches du régime de Ben Ali. À l'appui de cette affirmation, l'AMT a insisté sur la présence au sein du SMT de Khaled Abbès, l'un des auteurs du coup de force de 2005 à l'AMT, ainsi que celle de l'ancien premier président de la Cour de cassation, Mohamed Ellejmi, figure de la hiérarchie judiciaire acquise au régime de Ben Ali 37.

Dans le paysage des organisations de magistrats, l'UMA représente les juges administratifs. Ce syndicat trouve son origine dans l'éviction en septembre $2011 \mathrm{du}$ $1^{\mathrm{er}}$ président du tribunal administratif nommé en 2007 sous Ben Ali. Une partie des magistrats administratifs s'est élevée contre la nomination d'une nouvelle $1^{\text {re }}$ présidente et a créé une commission de protection de l'indépendance du tribunal administratif qui, en octobre 2011, s'est transformé en UMA attirant avec lui un peu plus d'une cinquantaine d'adhérents sur un corps comprenant alors une centaine de magistrats 38 . De leur côté, les soutiens à la nouvelle $1^{\text {re }}$ présidente rejoignent les rangs de l'AMT. Par conséquent, sans surprise, on constate que l'UMA agit la plupart du temps de concert avec le SMT et, par conséquent, souvent contre l'AMT 39.

Les différentes conceptions de la fonction judiciaire véhiculées par les organisations professionnelles se reflètent sur leur vision respective du CSM. L'AMT est porteuse d'un modèle d'autogouvernement de la magistrature dont précisément le CSM est le principal outil. Conséquence logique, ce dernier doit avoir de larges compétences dans l'administration de l'organisation juridictionnelle, à l'image des CSM des pays du Nord de l'Europe.

Il convient donc d'accroître les attributions du CSM dans le champ de la supervision de l'administration des tribunaux et de leur mode d'organisation administrative et financière. Pour éviter que l'inspection générale des services juridictionnels ne se

36. Ibid., p. 180.

37. Mohamed Sala BEN AïssA, «Pouvoir judiciaire et transition politique en Tunisie », in Éric GoBE (dir.), Des justices en transition dans le monde arabe? Contributions à une réflexion sur les rapports entre justice et politique, op. cit., p. 117.

38. Entretien avec Ahmed Souab, membre fondateur de 1'UMA, 22 mars 2018.

39. On notera l'absence des magistrats financiers dans les débats autour du CSM. Selon Ahmed Souab, ces derniers exercent dans un domaine très technique qui ne les met ni en situation de confrontation avec le pouvoir politique ni en position de revendiquer. Ibid. 
transforme en un instrument de répression de l'exécutif, elle doit être rattachée au CSM. Quant à la question de la composition du tiers de non-magistrats au sein des trois conseils de justice, l'AMT affiche clairement sa réticence à la présence d'avocats en leur sein. Considérant que ces derniers sont amenés en raison de leur fonction judiciaire à intervenir dans la vie professionnelle du juge, ils pourraient très bien utiliser leur qualité de membre du CSM pour exercer des pressions sur les magistrats. C'est pour des raisons du même ordre que l'AMT exige que le conseil de discipline soit composé uniquement de juges 40 .

La vision des attributions du CSM développée par le SMT est moins maximaliste que celle de l'AMT, sans en être très éloignée. Le syndicat ne voit pas d'inconvénients à la présence d'avocats dans le tiers composé de non-magistrats à condition que ces derniers n'exercent plus pendant leur mandat au sein du CSM afin d'éviter tout conflit d'intérêts. Le SMT soutient le même raisonnement concernant leur éventuelle intégration dans le conseil de discipline pour la même raison 41 .

À la différence de l'AMT, le syndicat ne souhaite pas que l'inspection des affaires juridictionnelles soit rattachée au CSM, là-aussi pour des raisons relatives aux conflits d'intérêts. Autrement dit, il n'est pas bon qu'une institution comme le CSM puisse «s'inspecter lui-même » et, par conséquent, s'occuper de l'administration des juridictions. Aussi propose-t-il de dédoubler l'inspection : une première resterait sous la tutelle du ministère de la Justice, tandis que la seconde serait une autorité publique indépendante 42 .

Sur cette question, l'UMA exprime la position la plus radicale. Il convient de se débarrasser de l'inspection au motif que la justice administrative n'y a jamais été soumise et qu'elle pourrait se transformer en un instrument aux mains de l'exécutif pour remettre en cause l'indépendance de la Justice. L'autre spécificité de l'UMA est de revendiquer, au nom d'une égale compétence des magistrats dans leurs ordres respectifs, l'égalité numérique des trois conseils 43 . Sur les deux autres points principaux du débat (la composition du conseil de discipline, ainsi que celle du tiers des non-magistrats et de la place des avocats en son sein), l'UMA tient le même discours que le SMT. Si leur position n'est pas aussi radicale que l'AMT vis-à-vis du barreau, leurs propositions reviennent à tenir les avocats en lisière du CSM.

Les positions divergentes entre représentants de la magistrature et de l'avocature vis-à-vis du CSM sont révélatrices de la lutte à laquelle se livrent les deux principaux corps de l'institution judiciaire pour imposer leur conception légitime de la justice. Dans un contexte politique volatil, il s'agit alors pour l'ONAT et les organi-

40. Entretien avec l'ancienne et le nouveau président de l'AMT Raoudha Karafi et Hmedi Anas, 21 mars 2018.

41. Entretien avec Raoudha Laabidi, ancienne présidente du SMT, 19 mars 2018.

42. $\mathrm{PV} \mathrm{n}{ }^{\circ} 13$, Séance de la commission de législation générale (en arabe), 25 mars 2015, ARP.

43. Entretien avec Walid Helali, président de l'UMA, 26 mars 2018. Le projet du ministère de la Justice prévoit une répartition inégale des membres entre le conseil de la justice judiciaire (27) et les conseils administratif (21) et financier (21). Cette différence est justifiée par le ministre de la Justice en raison de l'écrasante domination numérique des magistrats de l'ordre judiciaire (environ 2200 ) sur ceux des ordres administratif et financier (un peu plus d'une centaine chacun). 
sations de magistrats de se positionner comme des acteurs de la redéfinition du fonctionnement de la justice.

\section{Un texte contre le corporatisme des magistrats ?}

Après l'installation du Gouvernement issu des élections législatives de 2014, le nouveau ministre de la Justice, Mohamed Salah Ben Aïssa, marque de son empreinte le nouveau projet de loi gouvernemental : il reprend à son compte certaines remarques exprimées par les magistrats de l'AMT 44 tout en se montrant sensible à l'argumentaire constitutionnel de l'ONAT. S'il exclut que les avocats représentent seuls les indépendants cités dans l'article 112, son texte prévoit que le tiers des non-magistrats soit constitué d'une majorité d'avocats et d'une minorité de professeurs de droit.

Ce dernier point suscite l'ire de l'AMT. L'association en appelle également aux standards internationaux pour refuser la formulation de l'article $1^{\text {er }}$ de ce deuxième avant-projet gouvernemental, qui a fait disparaître du texte initial l'expression « le CSM représente le pouvoir juridictionnel » et l'a remplacée par « le CSM jouit de la personnalité juridique » 45 . L'AMT inaugure alors le temps des actions collectives en appelant à une grève des audiences dans les tribunaux.

Face aux pressions exercées par l'AMT, le ministre de la Justice accepte d'amender l'article $1^{\text {er }}$ qui retrouve sa forme initiale, mais il refuse de revenir sur la composition du tiers des non-magistrats indépendants, au motif que la présence des avocats est liée à leur statut constitutionnel et que les universitaires formant en amont les autres juristes ont le recul et les compétences nécessaires à un exercice indépendant de leurs fonctions au sein du CSM 46.

Approuvé en conseil des ministres, le projet de loi gouvernemental est transmis le 12 mars à la commission de législation générale de l'ARP où les députés doivent examiner le texte. Cette dernière est alors composée de parlementaires hostiles au texte gouvernemental. Tout d'abord, elle est le pré carré des députés avocats (12 des 21 membres) dont les positions sont similaires à celles de l'ONAT 47 . Ensuite, en termes d'affiliation politique les parlementaires de Nidaa Tounes 48 et du parti isla-

44. Universitaire, spécialiste de droit public, Mohamed Salah Ben Aïssa fait partie d'une élite de juristes, bilingue arabe-français, imprégnée de la culture juridique libérale et des droits de l'homme. Il adhérait à une vision élargie des attributions du CSM, tout en évitant de s'aligner complètement sur les positions de l'AMT afin de ne pas susciter une réaction de rejet du Gouvernement et des parlementaires. Entretien avec l'ancien ministre de la Justice, 7 novembre 2017.

45. L'UMA soutient la position inverse et considère que la personnalité morale doit être conférée au CSM pour des raisons procédurales. Entretien avec Ahmed Souab, op. cit.

46. Entretien avec l'ancien ministre de la Justice, op. cit.

47. De surcroît les avocats, comme sous d'autres cieux, mettent en avant leurs compétences de juristes «pour délégitimer les prétentions concurrentes à intervenir dans le débat [et] pour discuter des politiques de la justice » (Antoine VAUCHEZ et Laurent WILLEMEZ, La justice face à ses réformateurs (1980-2006), Paris : PUF, 2007, p. 94). Par ailleurs, l'avocature est la profession la plus représentée au sein de l'ARP (33 membres, soit $15 \%$ des parlementaires) devant les enseignants du secondaire $(11 \%)$.

48. L'Appel de la Tunisie, parti fondé par l'ancien président de la République, feu Béji Caïd Essebsi, est un rassemblement hétéroclite qu'unit, au moment de sa naissance, le rejet du parti Ennahdha : on y trouve alors des figures politiques proches de Habib Bourguiba, des caciques du parti dissous du président déchu, des patrons inquiets pour la bonne marche de leurs affaires, mais aussi des anciens militants de gauche. 
miste Ennahdha y sont massivement représentés (70\%). Or, le premier parti, qui a remporté les élections législatives de 2014 (mais pas la majorité absolue des sièges), est dominé par des élites issues du régime autoritaire de Ben Ali. Ces dernières sont marquées par le «référentiel régalien » 49 d'une fonction judiciaire subordonnée à l'exécutif, tandis que les cadres d'Ennahdha, réprimés sous les présidences de Ben Ali et Bourguiba, se méfient d'une "magistrature qui (pour partie) a été le bras nécessaire de cette justice répressive » 50 et sur laquelle ils souhaitent exercer un certain contrôle 51 .

\section{II.1. Des magistrats et des auxiliaires de justice malmenés}

La commission de législation générale va détricoter le projet gouvernemental, en affirmant la nécessité pour le pouvoir juridictionnel de rendre des comptes aux « représentants de la Nation ». L'articulation du corporatisme des représentants du barreau avec la logique politique de parlementaires réticents à donner trop de compétences au CSM débouche sur la présentation à l'assemblée plénière d'un projet de loi fort éloigné de sa version initiale.

Les magistrats, notamment ceux de l'AMT, sont dans le collimateur des parlementaires. Le président du groupe Nidaa Tounes à l'ARP, lui-même avocat d'affaires, proclame que le projet de CSM présenté par l'AMT est « effrayant » car instituant « un gouvernement des juges ». Il s'en prend au fait que l'AMT propose un conseil de discipline exclusivement composé de juges. Et les membres de la commission d'interpeler l'AMT sur la corruption de la magistrature et les dangers de voir des magistrats sans contrôle faire preuve de laxisme à l'égard de collègues fautifs 52 .

Alors que l'AMT allègue que les standards internationaux vont dans le sens d'une fermeture du conseil de discipline aux non-magistrats, la commission de Venise affirme le contraire et conforte ainsi le positionnement des membres de la commission 53. Quant au projet gouvernemental, s'il prévoit une composition mixte du conseil de discipline ( 5 juges et un non-magistrat), il ne permet pas aux membres extérieurs à la magistrature de participer au vote. Arguant du caractère inconstitutionnel de cette disposition, l'ensemble de la commission s'est mis d'accord pour

49. Nadia Bernoussi et Abderrahim El MASLOUHI, «Les chantiers de la "bonne justice". Contraintes et renouveau de la politique judiciaire au Maroc », Revue française de droit constitutionnel (RFDC), 91, 2012, p. 479-510.

50. Jean-Philippe BRAS, « De l'État légal à l'État de droit? Le statut constitutionnel de la justice au Maghreb », op. cit., p. 91.

51. Notamment à travers les nominations au CSM : lors de la discussion à l'ANC du chapitre V de la constitution relatif au pouvoir juridictionnel, Ennahdha a proposé un amendement, voté puis retiré, prévoyant que «les nominations aux hautes fonctions judiciaires se font par décret gouvernemental sur proposition du ministre de la Justice ».

52. $\mathrm{PV} \mathrm{n}^{\circ} 11$, Séance de la commission de législation générale, 23 mars 2015.

53. Pour éviter la prégnance d'une vision corporatiste à ce sujet, la commission de Venise, non seulement préconise de renforcer la présence des membres non-magistrats au sein du conseil de discipline, mais aussi d'en faire une instance paritaire. $\mathrm{PV} \mathrm{n}^{\circ} 15$, Séance de la commission de législation générale, 30 mars 2015. 
que chacun des trois conseils du CSM statue sur la discipline en leur formation complète 54 .

La composition mixte du conseil de discipline est revendiquée explicitement de la part des avocats comme une forme de contrôle de la part des non-magistrats : il s'agit de créer un équilibre au sein du CSM et, par conséquent, le tiers des nonmagistrats doit se voir attribuer un rôle effectif dans la prise de décision 55.

Si la commission a, en règle générale, repoussé les propositions de l'AMT, elle s'est montrée à peine plus compréhensive à l'égard de certaines de celles formulées par l'UMA et le SMT : elle récuse l'idée d'élever l'inspection des affaires juridictionnelles au statut d'autorité publique indépendante tout comme celle consistant à la faire disparaître. D'ailleurs, elle exclut des attributions du CSM l'inspection générale des affaires juridictionnelles et l'Institut supérieur de la magistrature 56 . Toutefois, elle reprend à son compte la proposition de l'UMA relative à l'égalité numérique des trois conseils : la commission décide que chaque conseil sera constitué de 15 membres. Mais ce faisant, elle change de manière drastique le ratio entre magistrats élus et magistrats nommés au détriment des premiers, ce qui suscite la désapprobation de l'AMT, du SMT et même de l'UMA 57.

La composition du CSM mécontente également les autres professions juridiques (les huissiers de justice, les notaires, les experts judiciaires, etc.) qui, lors des auditions devant la commission, revendiquent leur intégration dans la catégorie des nonmagistrats. Mais leur demande se heurte à une fin de non-recevoir de la part des avocats de la commission qui aspirent à dominer l'espace juridique. Les rappels lors des débats de la différence entre des avocats, «partenaires » à égalité avec les magistrats «dans la réalisation de la justice », et les autres « auxiliaires de justice »statut dont s'est débarrassée la profession avec la chute du régime de Ben Ali viennent délégitimer les revendications des professions juridiques concurrentes des avocats. La commission reprend à son compte la composition du tiers de nonmagistrats telle que mentionnée dans le texte gouvernemental : ne peuvent être membres que les avocats, les universitaires et les experts comptables (pour le conseil de la magistrature financière). Elle exclut l'éventuel élargissement aux huissiers et aux experts judiciaires qui, malgré le caractère libéral de leur exercice professionnel,

54. COMMISSION DE LA LEGISLATION GENERALE, Rapport concernant le projet de loi organique $n^{\circ}$ 16/2015 relatif au Conseil Supérieur de la Magistrature (en arabe).

55. «Début des sessions d'audition. La commission de législation générale achève la discussion générale autour de la loi sur le CSM », Le Maghreb, 18 mars 2015.

56. Rapport de la commission de la législation générale concernant le projet de loi organique, $n^{\circ} 16 / 2015$, op. cit.

57. Le projet du ministère de la Justice prévoit au total 10 magistrats nommés ex officio et 36 élus, contre 12 magistrats ex officio et 18 élus après amendement du texte par la commission. L'UMA préconisait pour chaque conseil 4 magistrats ex officio et 8 élus (soit un ratio deux-tiers élus et un tiers nommé). De son côté, le SMT préconisait une diminution du nombre de membres du conseil de la magistrature judiciaire à 21 , mais également des autres conseils à proportion, de manière à garder la prépondérance numérique du premier. Entretien avec Raoudha Laabidi, op. cit. 
sont assignés à leur positionnement infériorisé dans la division juridique du travail 58 .

\section{II.2. Un CSM aux attributions rognées}

Les amendements introduits par la commission rejoignent des propositions formulées par l'ONAT. Le travail de réécriture des dispositions générales du projet gouvernemental par la commission (notamment l'article $1^{\text {er }}$ ) contribue encore à élargir le fossé entre l'AMT et la commission. Cette dernière a remplacé les expressions « le CSM est une institution constitutionnelle qui représente le pouvoir juridictionnel » et «le CSM garantit l'indépendance du pouvoir juridictionnel et le bon fonctionnement de la justice » par le «CSM est une institution constitutionnelle qui garantit le bon fonctionnement de la justice et son indépendance ». Par conséquent, exit la notion de « pouvoir juridictionnel » 59 .

Face à cette nouvelle rédaction de l'article $1^{\mathrm{er}}$, ajoutée à l'intégration d'un représentant de la magistrature militaire au sein du conseil de la justice judiciaire, à la restriction des compétences du CSM et à sa composition, la présidente de l'AMT évoque, lors d'une conférence de presse, un « coup d'État de la commission contre la Constitution » 60. Le bureau exécutif de l'AMT lance un appel à la grève des audiences pour les 28 et 29 avril dans tous les tribunaux de Tunisie, mais il n'est ni suivi par l'UMA, qui le considère comme improvisé et unilatéral, ni par le SMT, qui trouve prématuré de conduire une action, alors que le texte n'a pas encore été soumis à la plénière de l'ARP.

La veille de l'ouverture des travaux à l'assemblée plénière, l'Instance provisoire de supervision de la justice judiciaire (IPSJJ), CSM provisoire créé en 2013 61, publie un communiqué et tient une conférence de presse, au cours de laquelle elle reprend largement les critiques formulées par l'AMT : le texte revu par la commission «porte atteinte aux standards internationaux concernant l'indépendance de la magistrature, notamment en ne faisant plus référence au pouvoir juridictionnel »; il contribue à « amoindrir le rôle du CSM dans la conduite de la carrière des magistrats » et de « la discipline » en maintenant l'inspection des affaires juridictionnelles sous la supervision du ministère de la Justice ; il « contrevient à l'article 112 de la Constitution en incorporant la magistrature militaire au sein du CSM »; enfin, l'IPSJJ

58. PV $\mathrm{n}^{\circ} 18$, Séance de la commission de législation générale, 7 avril 2015, ARP. Sur les processus de constitution de hiérarchies dans l'espace juridique, voir Laurent WILLEMEZ, Des avocats en politique (1840-1880). Contribution à une sociohistoire de la profession politique en France, thèse pour le doctorat de science politique, Université de Paris 1, 2000.

59. Rapport de la commission de la législation générale concernant le projet de loi organique, $n^{\circ} 16 / 2015$, op. cit.

60. «L'AMT : ce qui se passe au sein de la commission générale est un coup d'État contre la Constitution », Le Maghreb, 24 avril 2015.

61. Cette instance était chargée de statuer « sur la carrière professionnelle des magistrats » et « d'émettre un avis consultatif sur les projets de loi relatifs au fonctionnement de la justice » et à la « réforme de la justice judiciaire » (article 2). JORT, «Loi organique $n^{\circ} 2013-13$ du 2 mai 2013, relative à la création d'une instance provisoire pour la supervision de la justice judiciaire », 7 mai 2013, p. 1410-1413. 
dénonce le fait que le texte ne lui a pas été transmis « comme le dispose la loi » 62 . Il est vrai que l'IPJJ est composée de quinze magistrats (sur vingt membres) majoritairement adhérents à l'AMT (le SMT a boycotté l'élection), dont la porte-parole Wassila Kaabi est une figure emblématique de la « résistance judiciaire » 63 à l'autoritarisme, au début des années 2000 .

En revanche, les représentants du barreau expriment leur satisfaction face à un texte qu'ils considèrent comme globalement conforme à la Constitution, car consacrant l'indépendance de la justice, une composition mixte du CSM, ainsi que le principe du procès équitable 64 .

Les travaux de l'Assemblée plénière débutent le 12 mai, alors qu'une partie des magistrats, à l'appel de l'AMT, sont en grève et manifestent devant le Parlement. Dans l'hémicycle, les revendications des magistrats sont soutenues par... le ministre de la Justice (tout au moins implicitement) 65 et par les deux formations politiques les plus aux antipodes de l'échiquier politique : Afak Tounes (huit députés) et le Front populaire (FP) 66 (quinze députés). Le premier parti d'obédience économique libérale, membre de la coalition au Gouvernement, composé principalement de membres des professions libérales et d'hommes d'affaires a déjà, par l'intermédiaire de sa représentante au sein de la commission, refusé de voter certains amendements proposés par la commission au motif qu'ils étaient contraires à la Constitution. Le parti reprend à son compte l'essentiel de l'argumentaire de l'AMT, notamment à propos de la nécessaire supervision de l'inspection des affaires juridictionnelles par le CSM et autour du refus de voir les avocats participer aux décisions du conseil de discipline.

Quant au FP, il se retire un moment des débats pour signifier son désaccord sur la disparition dans l'article $1^{\text {er }}$ de toute référence au « pouvoir juridictionnel ». Après d'âpres négociations, il obtient une nouvelle rédaction dudit article. Il fait également

62. , «L'IPSJJ : "La commission de législation générale a dépouillé le CSM de ses missions constitutionnelles" », Le Maghreb, 12 mai 2015.

63. Sur cette notion, voir Liora ISRAËL, Robes noires, années sombres. Avocats et magistrats en résistance pendant la Seconde Guerre mondiale, Paris : Fayard, (2005). En mars 2005, Wassila Kaabi était membre du bureau exécutif de l'AMT qui avait publié un communiqué de soutien du barreau après l'arrestation de l'avocat opposant au régime de Ben Ali, Mohamed Abbou. Le texte rédigé par l'AMT dénonçait « les atteintes à l'inviolabilité du tribunal ». Il exprimait également sa solidarité avec des avocats « gravement molestés par les forces de sécurité » et «empêchés d'exercer leur profession » (Sana BEN ACHOUR, «La féminisation de la magistrature en Tunisie entre émancipation féminine et autoritarisme politique », op. cit., p. 71). Tout comme le président de l'AMT de l'époque, Ahmed Rahmouni, elle a été sanctionnée par le régime de Ben Ali qui lui a imposé une mutation contrainte à Gabès à $420 \mathrm{~km} \mathrm{de}$ Tunis, son lieu d'exercice professionnel initial. Entretien avec Wassila Kaabi, 22 mars 2018.

64. «L'ONAT présente sa vision du projet de loi sur le CSM : c'est un projet constitutionnel... Il consacre l'indépendance de la justice, mais il convient d'y introduire quelques amendements », Le Maghreb, 13 mai 2015.

65. Celui-ci sera limogé du Gouvernement en octobre 2016, à la suite d'un désaccord avec le chef du Gouvernement qui a apporté son soutien à la version amendée par les députés en commission et en plénière.

66. Le Front populaire est une coalition politique regroupant associations et organisations partisanes, allant de l'extrême gauche (dont le Parti des travailleurs et le Parti unifié des patriotes démocrates sont les composantes principales) aux nationalistes arabes en passant par les écologistes. Deux de ses dirigeants (Choukri Belaïd et Mohamed Brahmi) ont été assassinés en 2014 par de supposés salafistes-djihadistes. 
passer quelques amendements allant dans le sens d'un très relatif élargissement des attributions consultatives du CSM et plus particulièrement de son assemblée plénière. Mais pour autant la position du FP reste la même : le projet de loi ne garantit pas l'indépendance de la justice et, par conséquent, il est hors de question pour le groupe parlementaire de le voter.

En revanche, les deux principaux partis politiques du Parlement s'estiment plutôt satisfaits du projet de loi déposé au bureau de l'ARP, même si Ennahdha déclare par la voix de la vice-présidente de la commission, Latifa Habachi, que son parti a l'intention d'amender le texte pour remplacer le représentant de la magistrature militaire par le procureur général de la République, directeur des administrations judiciaires au sein du conseil de la magistrature judiciaire 67. Après négociations avec Nidaa Tounes, le mouvement islamiste accepte la présence du représentant de la justice militaire à condition qu'une disposition transitoire prévoie son remplacement temporaire par le procureur général, directeur des services judiciaires, dans l'attente du vote d'un amendement des lois relatives aux tribunaux militaires. Par ailleurs, Nidaa Tounes et Ennahdha sont d'accord pour modifier à la marge la composition du tiers de non-magistrats du conseil de la magistrature judiciaire en remplaçant un avocat par un huissier de justice. La veille du vote, conscientes que la partie est largement jouée, les organisations de magistrats, pour une fois réunies, publient un communiqué dans lequel elles demandent « aux présidents de la République et de l'ARP, au chef du Gouvernement, ainsi qu'à tous ceux qui défendent l'indépendance de la justice de renvoyer le texte devant l'Instance provisoire de contrôle de la constitutionnalité des projets de loi (IPCCPL) en raison de son inconstitutionnalité » 68 . Effectivement, le lendemain, l'ARP approuve le projet de loi. Pour autant, ce vote ne signifie pas que le texte est arrivé à bon port, les députés du FP réunissant 30 signatures afin de soumettre le texte à l'IPCCPL.

La décision de l'IPCCPL de déclarer inconstitutionnelles certaines dispositions de la loi conforte l'AMT et le ministre de la Justice dans leurs prises de position. Bien que 1'IPCCPL soit loin d'avoir accepté tous les recours, elle sanctionne, entre autres, la remise en cause partielle de la composition du conseil de la justice judiciaire qui apparaît comme la censure la plus dérangeante pour les députés. La présence d'un représentant de la magistrature militaire est considérée comme inconstitutionnelle, tout comme l'absence d'un enseignant-chercheur universitaire dans le tiers des non-magistrats du conseil judiciaire 69. In fine, après avoir connu de nombreuses pérégrinations et avoir été de nouveau censuré par l'IPCCPL, le projet gouvernemental est voté par l'ARP le 23 mars 2016.

La dynamique d'élaboration chaotique de la loi sur le CSM a constitué un moment de tension entre les représentants des professions juridiques, un ministre de la Jus-

67. Ennahdha exprime ainsi ses réserves vis-à-vis d'une justice militaire qui a été l'instrument de la répression du mouvement islamiste au début des années 1990.

68. «Après l'adoption du projet de loi relatif au CSM : toutes les organisations professionnelles suspendent leur participation aux commissions de rédaction du ministère de la Justice et demandent au Premier ministre de renvoyer le texte devant l'IPCCPL », Le Maghreb, 16 mai 2015.

69. «IPCCPL, Décision $n^{\circ}$ 2015/02, du 8 juin 2015 se rapportant au projet de loi relatif au CSM », JORT, 12 juin 2015, p. 1495. 
tice guère soutenu par le chef du Gouvernement et des parlementaires majoritairement partisans d'un CSM aux compétences restreintes. Depuis fin 2016, avec le processus de mise en place du CSM, cette dynamique institutionnelle hautement conflictuelle perdure, toujours alimentée par les interactions entre le jeu des structures professionnelles et celui de l'arène politique.

\section{Conclusion}

L'examen du processus d'élaboration et d'adoption de la loi relative au CSM montre combien les enjeux professionnels de l'arène judiciaire peuvent être hautement conflictuels et politisés. De manière générale, il a mis en exergue le fait que les débats et actions des professionnels du droit ont pris place dans un « espace carrefour » où les avocats ont joué « des stratégies continues de double jeu». Ils ont su convertir des ressources politiques en capital juridique et « des ressources juridiques en capital de légitimité politique » 70 .

Depuis 2011, à la différence des magistrats dont le statut interdit tout engagement direct en politique, la profession d'avocat en Tunisie a vu certaines de ses composantes investir l'arène politique officielle, notamment le Parlement, et a su tirer profit des actions collectives menées sous la houlette de sa fraction militante et des instances ordinales tant sous Ben Ali que pendant la période de soulèvement populaire (décembre 2010 - janvier 2011). Ces gains, bien que parfois remis en cause par les organisations de magistrats ont été utilisés comme des ressources pour accroître l'autonomie de la profession. Avec le processus « révolutionnaire » et de «transition démocratique », les revendications du barreau sont apparues d'autant plus légitimes que les valeurs véhiculées par la majeure partie des avocats étaient congruentes avec les changements politiques. Autrement dit, les avocats ont pu se prévaloir d'une « légitimité normative » 71 .

Instrument de la justice coercitive des régimes autoritaires de Bourguiba et de Ben Ali, la magistrature partait, de ce point de vue, avec un handicap. Toutefois, dans le cadre d'une alliance objective AMT-SMT-UMA, les représentants de la magistrature ont souvent su, dans la conduite de leur action professionnelle, faire prévaloir auprès des gouvernants leurs intérêts au détriment de ceux des avocats 72 . Ils ont, dans le même temps, été capables, de concert avec l'ONAT, lors des débats à l'ANC concernant le chapitre relatif au pouvoir juridictionnel, de construire une légitimité morale en se posant comme les défenseurs de l'indépendance de la justice

70. Sara DeZAlay, «Les juristes en Afrique : entre trajectoires d'État, sillons d'empire et mondialisation », article cité, p. 14

71. Celle-ci « repose sur la congruence entre les valeurs qu'elle promeut et les normes d'action qui guident la société dans son ensemble [...]. Elle repose sur l'idée que le critère de l'efficacité ne suffirait pas à rendre une profession légitime, si les finalités qu'elle poursuit ne sont pas en accord avec les valeurs d'une société » (Patrice DuRAN et Thomas LE BIANIC, «Introduction générale », in Thomas LE BIANIC et Antoine VION (dir.), Action publique et légitimités professionnelles, op. cit., p. 29).

72. Éric GoBE, «Les mobilisations professionnelles comme mobilisations politiques : les avocats tunisiens de la "révolution" à la "transition" », in Choukri HMED et Laurent JEANPIERRE (dir.), dossier « Révolutions et crises politiques : Maghreb/Machrek », Actes de la recherche en sciences sociales, 211-212, 2016, p. 93-107. 
contre des constituants ayant des velléités d'assujettir la justice. Ce faisant, sur ce point, ils ont donné raison à l'une des hypothèses formulées par Lucien Karpik et Terence C. Halliday selon laquelle plus les avocats et les magistrats se soutiennent dans un projet libéral, plus ils sont efficaces pour créer les conditions de l'émergence d'un État de droit.

Toutefois, l'épisode de l'élaboration de la loi sur le CSM pourrait laisser penser que le «complexe juridique» fonctionne à front renversé : les magistrats défendraient, à travers un CSM aux compétences élargies, une justice émancipée du pouvoir politique, alors que les avocats seraient, à l'opposé, pusillanimes souhaitant avoir affaire à un ministère de la Justice disposant de moyens de contrôle de la magistrature et continuant à administrer l'essentiel des services judiciaires.

Nous l'avons vu, cette lecture est biaisée, mais elle montre a contrario que l'approche en termes de « complexe juridique » mériterait d'être complexifiée... La dynamique de ce complexe ne peut se comprendre indépendamment de la prégnance du référentiel régalien chez une partie des élites politiques. Le passé proche rend la revendication d'autonomie professionnelle de la magistrature suspecte aux yeux du barreau, d'une partie des parlementaires et de l'Exécutif (structuré autour d'un compromis passé entre des élites de l'ancien régime et le parti Ennahdha) : elle ne serait que l'expression des magistrats d'une volonté de s'affranchir de tout contrôle politique.

Les conflits autour de la nouvelle loi relative au CSM montrent qu'en Tunisie le «paradoxe redoutable d'une justice à nouveau asservie en raison de ses asservissements passés » 73 demeure d'actualité.

73. Jean-Philippe BRAS, «De l'État légal à l'État de droit? Le statut constitutionnel de la justice au Maghreb », op. cit., p. 91. 